\title{
Rosuvastatin-Induced Rhabdomyolysis, Pancreatitis, Transaminitis, and Acute Kidney Injury
}

\author{
Brent Wagner, MD, MS; G. Patricia Escobar, DVM; D. Bradley Jackson, DO; and Joshua DeAguero
Changing medications within a drug class requires considering the indication and dosage, possible adverse effects, and drug-drug interactions.

\author{
Author affiliations \\ can be found at the \\ end of the article. \\ Correspondence: \\ Brent Wagner \\ (brwagner@salud.unm.edu)
}

Fed Pract. 2021;38(suppl 4). Published online November 12. doi:10.12788/fp.0199
A ttention should be paid to changing a tolerated medication to another within its class. Many drugs approved by the US Food and Drug Administration (FDA), have equivalent therapeutic properties as existing drugs. Rarely do such medications share the same potency and adverse effect (AE) profile.

\section{CASE PRESENTATION}

A 77-year-old man presented to the emergency department (ED) at the Raymond G. Murphy Medical Center in Albuquerque, New Mexico, with a 1-month history of progressive muscle weakness, which was so severe that he required assistance rising from chairs. The symptoms began when he switched from atorvastatin $40 \mathrm{mg}$ daily to rosuvastatin $40 \mathrm{mg}$ daily. A nephrology consultation was requested for an elevated plasma creatinine.

The patient reported strict adherence to his prescribed medications. In the days following the switch to rosuvastatin, he noticed that his urine turned black. He described the color as "like burnt coffee." The color gradually cleared before his ED presentation. The patient stopped taking rosuvastatin the day prior to presentation and noted improvement of his symptoms. Review of symptoms was significant for lower extremity paresthesia and numbness the day he started rosuvastatin. He had no symptoms of decompensated heart failure and no recent exacerbations requiring alteration of his diuretic regimen.

The patient's medical history was significant for traumatic brain injury with complex partial seizures, carpal tunnel syndrome, dyslipidemia, coronary artery disease with percutaneous intervention to the right coronary artery in the late 1990s, atrial fibrillation and ventricular tachycardia, status post implant- able cardioverter defibrillator, heart failure with reduced ejection fraction (25\%) attributed to ischemic cardiomyopathy, hypertension, lower urinary tract symptoms/prostatism, and previous bladder cancer. In the mid-1960s, the patient served in the US Army and had been deployed to South Korea. After the service, he worked for the local city government. He was retired for about 15 years. He reported no tobacco, alcohol, or recreational drug use and no tattoos. He did not require prior blood or blood product transfusions. None of his family members-parents, siblings, or children-had any history of kidney disease.

The patient's outpatient medications included levetiracetam $750 \mathrm{mg}$ twice daily, melatonin $9 \mathrm{mg}$ at night, menthol 16\%/methylsalicylate $30 \%$ topically up to 4 times per day as needed, aspirin $81 \mathrm{mg}$ once daily, fish oil $1000 \mathrm{mg}$ twice daily, amiodarone $400 \mathrm{mg}$ twice daily, hydralazine $20 \mathrm{mg} 3$ times daily, isosorbide mononitrate $60 \mathrm{mg}$ daily, metoprolol succinate $100 \mathrm{mg}$ daily, and tamsulosin $0.4 \mathrm{mg}$ at night. His vital signs were stable: afebrile $\left(97.5^{\circ} \mathrm{F}\right)$, normocardic (74 beats per minute), normotensive (118/78 $\mathrm{mm} \mathrm{Hg}$ ), and normoxic ( $98 \%$ on room air). On examination, he appeared elderly, somewhat frail, and chronically ill but in no acute distress. Affect was pleasant and appropriate, attention was high, and his thought process was logical. He had sparse, grey scalp hair. Extraocular movements were intact. Oral mucosa was pink and moist. His back was nontender, and there was no costovertebral tenderness bilaterally. The patient was in no respiratory distress, with a slightly hyperresonant chest to percussion bilaterally, very faint inspiratory basilar crepitant rales (that cleared with repeat inspiration), and was otherwise clear to auscultation 
throughout. An outline of an implanted pacemaker was evident on the chest under his left clavicle, with a laterally displaced apical impulse. The rate was normal and the rhythm was regular. Upper extremities demonstrated papyraceous skin but without cyanosis, clubbing, or edema. Radial pulses were slightly diminished. He had no lower extremity edema.

His laboratory values are provided in Table 1. Kidney function was stable months prior to admission. Of note, the blood urea nitrogen and plasma creatinine were increased from his baseline up to 47 and $5.89 \mathrm{mg} / \mathrm{dL}$, respectively. The serum glutamic-oxaloacetic transaminase and serum glutamic pyruvic transaminase were $1051 \mathrm{U} / \mathrm{L}$ and $408 \mathrm{U} / \mathrm{L}$, respectively. Plasma amylase and lipase levels also were elevated, $230 \mathrm{U} / \mathrm{L}$ and $892 \mathrm{U} / \mathrm{L}$, respectively. Creatine kinase was 41,099 U/L. Urinalysis demonstrated a specific gravity of 1.017 , $\mathrm{pH}$ of 5, and a large amount of blood (92 red blood cells/high power field).

A 12-lead electrocardiogram demonstrated a sinus rhythm, PR interval of $0.20 \mathrm{~ms}$, narrow QRS with a leftward frontal axis deviation, R-transition between precordial leads V1 and $\mathrm{V} 2$, and flattening of the ST segments in III, V1-V3 (Figure 1). A portable chest X-ray demonstrated clear lung fields, no evidence of effusion in the costophrenic area. Ultrasonography was conducted at the time of the examination (Figure 2). The kidneys were smoothly contoured, each measuring $>10 \mathrm{~cm}$; there was an exophytic cyst on the left. Otherwise, the cortices, perhaps slightly echogenic, did not appear diminished. The bladder was not abnormally enlarged.

Rosuvastatin-induced rhabdomyolysis, pancreatitis, transaminitis, and drug-induced acute kidney injury were considered high among the diagnostic differentials. The 3-hydroxy3-methyl-glutaryl-CoA (HMG-CoA) reductase inhibitor was stopped, and he was prescribed an acute renal insufficiency diet. All laboratory parameters improved with this change (Figure 3). Two months after presentation (and with rosuvastatin added to his list of adverse reactions), all symptoms resolved and his plasma creatinine reached a nadir of $1.22 \mathrm{mg} / \mathrm{dL}$.

\section{DISCUSSION}

Statin-class drugs inhibit the HMG-CoA reductase (Table 2). Upregulation of low-density lipoprotein cholesterol (LDL-C) receptors in the
TABLE 1 Patient's Laboratory Results

\begin{tabular}{|c|c|c|c|}
\hline Characteristics & $\begin{array}{c}\text { Reference } \\
\text { Range, Adults }\end{array}$ & $\begin{array}{l}\text { Most Recent } \\
\text { Results Before } \\
\text { Presentation }\end{array}$ & $\begin{array}{c}\text { Results at } \\
\text { Initial } \\
\text { Consultation }\end{array}$ \\
\hline Hemoglobin, g/dL & $13.5-17.7$ & 12.0 & 11.5 \\
\hline Hematocrit, \% & $42.0-53.0$ & 36.4 & 37.1 \\
\hline White-cell count, $\mu \mathrm{L}$ & $4.0-11.0$ & 5.9 & 7.8 \\
\hline Platelet count, $\mathrm{K} / \mathrm{mm}^{3}$ & $150-400$ & 232 & 166 \\
\hline Sodium, mmol/L & $137-145$ & 138 & 138 \\
\hline Potassium, mmol/L & $3.4-4.8$ & 4.5 & 4.3 \\
\hline Chloride, $\mathrm{mmol} / \mathrm{L}$ & $98-107$ & 108 & 106 \\
\hline Carbon dioxide, $\mathrm{mmol} / \mathrm{L}$ & $20-31$ & 21 & 23 \\
\hline Urea nitrogen, $\mathrm{mg} / \mathrm{dL}^{\mathrm{b}}$ & $9-20$ & 27 & 41 \\
\hline Creatinine, $\mathrm{mg} / \mathrm{dL}^{\mathrm{b}}$ & $0.66-1.25$ & 1.87 & 5.22 \\
\hline Glucose, mg/dL ${ }^{b}$ & $74-99$ & 83 & 83 \\
\hline Calcium, mg/dL & $8.4-10.2$ & 8.8 & 9.1 \\
\hline Phosphorus, mg/dL & $2.5-4.5$ & 3.5 & not available \\
\hline Magnesium, mg/dL & $1.6-2.3$ & 2.1 & 2.1 \\
\hline Protein, g/dL & $6.3-8.5$ & 6.8 & 6.7 \\
\hline Albumin, g/dL & $3.5-5.0$ & 3.6 & 3.7 \\
\hline Bilirubin, total, mg/dL & $0.2-1.3$ & 0.7 & 1.2 \\
\hline Bilirubin, direct, mg/dL) & $0.0-0.4$ & 0.0 & 0.3 \\
\hline $\begin{array}{l}\text { Serum glutamic-oxaloacetic } \\
\text { transaminase, U/L }\end{array}$ & $17-59$ & 45 & 1318 \\
\hline $\begin{array}{l}\text { Serum glutamic pyruvic } \\
\text { transaminase, } U / L\end{array}$ & $\leq 50$ & 32 & 454 \\
\hline Amylase, U/L & $30-130$ & not available & 230 \\
\hline Lipase, U/L & $23-100$ & 30 & 892 \\
\hline
\end{tabular}

aSpecific to Raymond G. Murphy Veterans Affairs Medical Center in Albuquerque, New Mexico; determined by the patient population and the laboratory methods used.

${ }^{b}$ Conversion: urea nitrogen units to $\mathrm{mM} \times 0.357$; creatinine units to $\mu \mathrm{M} \times 88.4$; glucose units to $\mathrm{mM} \times 0.05551$.

liver result in increased LDL-C uptake and cholesterol catabolism. ${ }^{1}$ Prescribed inhibitors of the HMG-CoA reductase-statins-are known to reduce mortality due to cardiovascular disease (CVD). Much like any other pharmaceutical agent with any measurable potency, HMG-CoA inhibitors can have AEs. Statin therapy has been associated with pancreatitis. ${ }^{2}$ Muscle toxicity is a complication of HMG-CoA reductase inhibitors, and statin-associated symptoms are a leading cause of nonadherence. ${ }^{3}$ Rosuvastatin 
FIGURE 1 12-Lead Electrocardiogram Showing Frontal Left-Axis Deviation and R Transition After V1

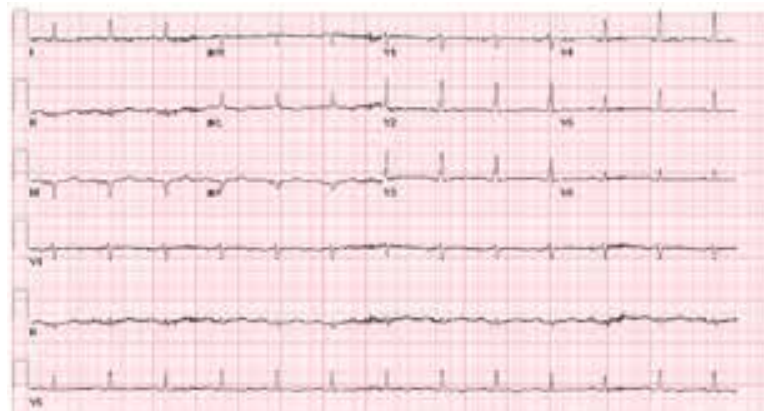

FIGURE 3 Improved Laboratory Results Following Rosuvastatin Discontinuation
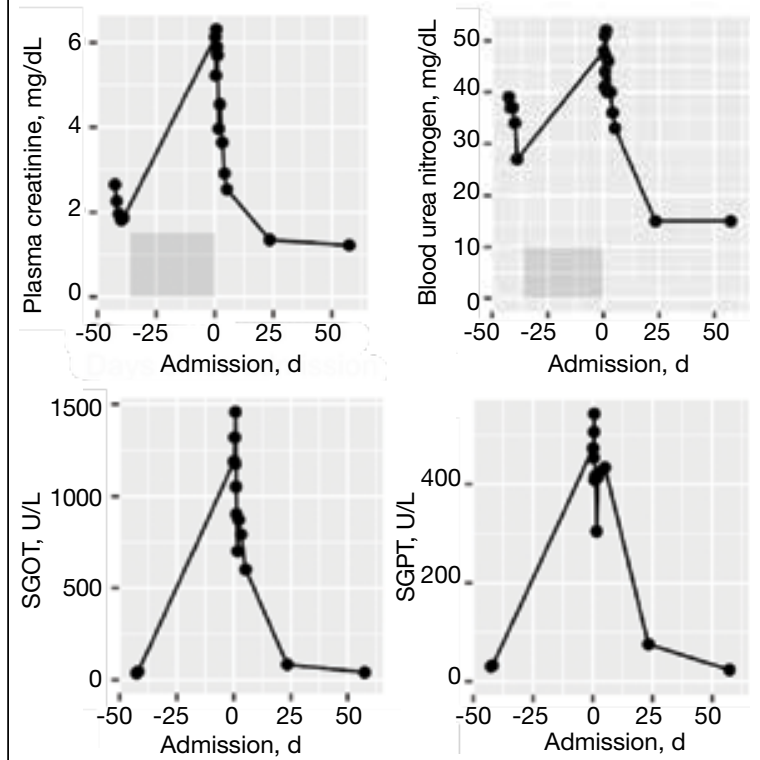

Abbreviations: SGOT, serum glutamic-oxaloacetic transaminase; SGPT, serum glutamic pyruvic transaminase.

had higher $\mathrm{AE}$ and drug reactions compared with that of atorvastatin and pitavastatin $(35.6 \%$, $8.7 \%$, and $22.2 \%$, respectively) in clinical trials for approval. ${ }^{4}$ We have reported concomitant adermatopathic dermatomyositis with statininduced myopathy in a 48-year-old man from simvastatin (40 to $80 \mathrm{mg}$ daily).

Toxin-induced myopathy should be considered early in the differential diagnosis of weakness. ${ }^{5}$ All HMG-CoA inhibitors have been associated with acute kidney injury, particularly at high doses and also are known to induce myopathies, sometimes with inclusion bodies. ${ }^{1}$ Muscle-related AEs correlate with the potency of an HMG-CoA reductase inhibitor according to an analysis using the FDA AE

\section{FIGURE 2 Bedside Kidney Ultrasonography}

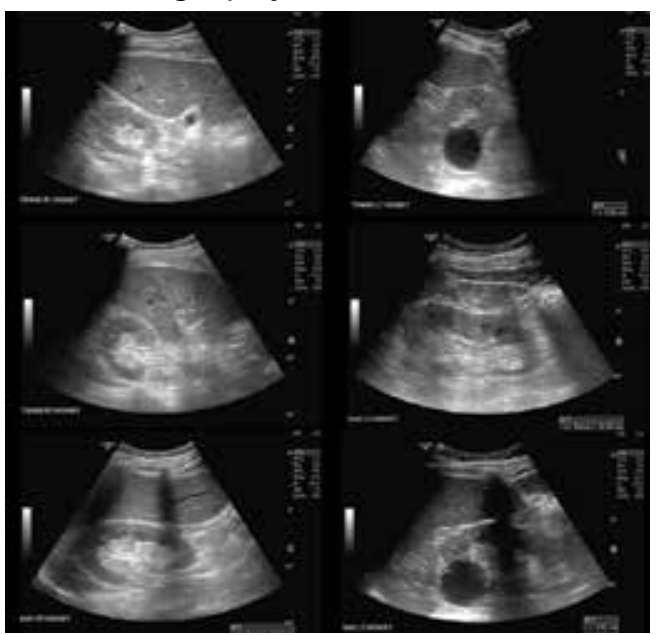

Both kidneys were $>10 \mathrm{~cm}$ in length and generally smoothly contoured without diminishment of cortical sizes. Of note, cysts (1 partially exophytic) in the left kidney; radiology noted normal liver size.

\section{FIGURE 4 Rosuvastatin Chemical} Structure

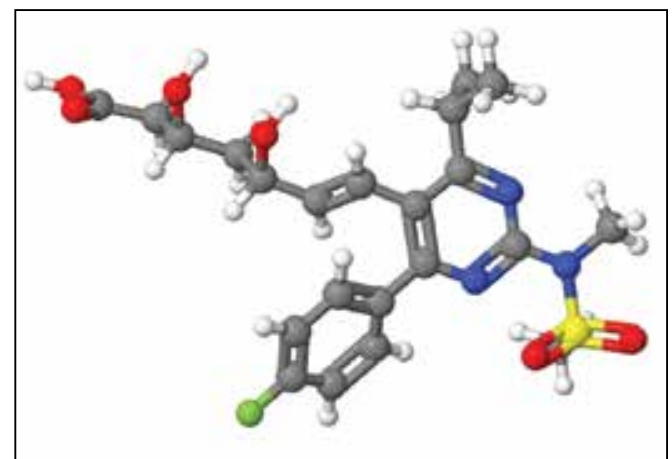

Source: Jmol, an open-source Java viewer for chemical structures in 3D (http://www.jmol.org).

Reporting System (AERS). ${ }^{6}$ Myalgia and rhabdomyolysis are well-known AEs of this class of medications. Furthermore, type II muscle atrophy - particularly in the proximal limb muscles - has been reported. ${ }^{5}$ Patients may have difficulty rising from chairs. ${ }^{1}$ Rosuvastatin had the strongest signal for muscular AEs (eg, myalgia, rhabdomyolysis, increased creatine phosphokinase level) from an FDA analysis of AERS. ${ }^{7}$

Rosuvastatin is the only HMG-CoA reductase inhibitor that causes dosedependent increases in proteinuria and hematuria (Figure 4). ${ }^{8}$ Rosuvastatin at a 5-mg dose may induce 4 times the proteinuria as a placebo. Typically, other statins potentially reduce proteinuria 
(without hematuria). Proteinuria may be induced by rosuvastatin even at low doses. ${ }^{8}$ Proteinuria is attributed to how rosuvastatin impacts proximal tubular function. ${ }^{9}$ The drug is transported into the proximal tubule by the organic anion transporter-3. Acute kidney injury has been associated with several statins, including rosuvastatin. 7,10 This may be associated with denuded tubular epithelia, active urinary sediment, acute tubular toxicity, vacuolated epithelial cells, and tubular cell casts. Unlike atorvastatin, the increase in proteinuria and hematuria also is dose dependent.

In patients with renal insufficiency (short of end-stage renal disease [ESRD]), most statins other than rosuvastatin are well tolerated and recommended for reduction of overall and CVD mortality risk. However, these benefits seem to diminish once ESRD is reached. Atorvastatin did not impact CVD mortality in patients with type 2 diabetes mellitus (T2DM) and ESRD (despite decreasing LDL-C). ${ }^{11}$ The AURORA study randomized $10 \mathrm{mg}$ of statin vs placebo in 2776 maintenance dialysis patients aged 50 to 80 years. Rosuvastatin lowered the LDL-C but did not affect all-cause mortality (13.5 vs 14.0 events per 100 patient-years). Patients randomized to rosuvastatin had more than twice as many unclassified strokes ( 9 vs 4). Rosuvastatin, although efficacious in reducing LDL-C, had no impact on CVD mortality, nonfatal myocardial infarction, or nonfatal stroke. ${ }^{12}$ Post hoc analysis demonstrated that in patients with T2DM with ESRD the hazard ratio for hemorrhagic stroke was 5.2. ${ }^{13}$

Rosuvastatin ranked lower than lovastatin, pravastatin, simvastatin, atorvastatin, and fluvastatin with respect to reduction of all-cause mortality in trials of participants with or without prior coronary artery disease. ${ }^{14} \mathrm{AEs}$, such as rhabdomyolysis, proteinuria, nephropathy, renal failure, liver, and muscle toxicity are higher with rosuvastatin than other medications in its class. ${ }^{15}$

\section{CONCLUSIONS}

For patients with existing CVD, standard clinical practice is to encourage increased and regular physical activity, cholesterollowering diets, weight loss, and smoking cessation. Hypertension should be treated. Glycemia should be well controlled in the setting of T2DM. $\beta$-blockers may be beneficial in those with histories of myocardial infarction
TABLE 2 Pharmacokinetic Parameters for HMG-CoA Reductase Inhibitors

\begin{tabular}{lccc} 
Statins (mg) & $\begin{array}{c}\text { Protein } \\
\text { Binding, \% }\end{array}$ & $\begin{array}{c}\text { Elimination } \\
\text { Half-Life, } \mathbf{~}\end{array}$ & $\begin{array}{c}\text { LDL Cholesterol } \\
\text { Reduction }\end{array}$ \\
\hline Atorvastatin (10-80) & $\geq 98$ & 14.0 & $38-54$ \\
\hline Fluvastatin (20-80) & 98 & 3.0 & $17-33$ \\
\hline Lovastatin (20-80) & $>95$ & $1.1-1.7$ & $29-48$ \\
\hline Pitavastatin (1-4) & $>99$ & 12.0 & $31-41$ \\
\hline Pravastatin (10-40) & 50 & 1.8 & $19-40$ \\
\hline Rosuvastatin (10-40) & 88 & 19.0 & $52-63$ \\
\hline Simvastatin (10-40) & 95 & 4.9 & $28-41$
\end{tabular}

Abbreviations: HMG-CoA, 3-hydroxy-3-methylglutaryl coenzyme A; LDL, low-density lipoprotein (plasma).

almmediate release, twice daily.

or heart failure with reduced systolic function. Statins are a valuable tool in the treatment of dyslipidemia.

Statin-induced muscle symptoms are a major reason for discontinuation and nonadherence. ${ }^{16}$ Statin-induced myalgia, myositis, and myopathy have been used interchangeably. ${ }^{17}$ Rhabdomyolysis, myalgia, increased creatine kinase, statin myopathy, and immunemediated necrotizing myopathy are among the clinical phenotypes caused by statins. ${ }^{17}$ There are 33,695 serious cases -1808 deaths-reported with rosuvastatin in the FDA AERS as of June 30, 2021. Myalgia, pain in extremity, muscle spasms, pain, and arthralgia top the list of AEs. When statin-induced symptoms occur, adherence is rarely improved by dismissive clinicians. ${ }^{18}$

Drugs in the same class often have common therapeutic properties. Potencies and $A E$ profiles are seldom uniform. The decision to add or change the brand of medication within a class should be balanced with considerations for the indication, duplications, simplification, AEs, appropriate dosage, and drug-drug interactions.

\section{Acknowledgments}

Brent Wagner is funded by a US Department of Veterans Affairs Merit Award (I01 BX001958), a National Institutes of Health R01 grant (DK-102085), Dialysis Clinic, Inc., and partially supported by the University of New Mexico Brain and Behavioral Health Institute (BBHI 2018-1008, 2020-21-002) and in part by the University of New Mexico's Signature Program in Cardiovascular and Metabolic Disease (CVMD); and the University of New Mexico School of Medicine Research Allocation Committee (C-2459-RAC, New Mexico 
Medical Trust). Brent Wagner is an Associate Member to the University of New Mexico Health Sciences Center Autophagy, Inflammation, and Metabolism Center of Biomedical Research Excellence (AIM CoBRE) supported by NIH grant P20GM121176.

\section{Funding}

National Institutes of Health Grant R01 DK-102085, Dialysis Clinic Inc., VA Merit Award 101 BX001958, Center for Integrated Nanotechnologies User Agreement 2019AU0120, Brain \& Behavioral Health Institute (grants 2018-1008, 202021-002), University of New Mexico's Signature Program in Cardiovascular and Metabolic Disease (CVMD), the University of New Mexico School of Medicine Research Allocation Committee (C-2459-RAC, New Mexico Medical Trust) and a metabolomics voucher from the AIM Center (NIH P20GM121176).

\section{Author affiliations}

Brent Wagner is Associate Chief of Staff for Research and Development; Patricia Escobar is a Research Technician (WOC); Bradley Jackson is an Internal Medicine Resident; and Joshua DeAguero is a Graduate Student (WOC); all at New Mexico Veterans Administration Health Care System, in Albuquerque. Brent Wagner is Director, Patricia Escobar is a Research Scientist, and Joshua DeAguero is a Biomedical Sciences PhD student; all at the Kidney Institute of New Mexico, University of New Mexico Health Science Center. Brent Wagner is an Associate Professor of Medicine; Patricia Escobar is a Research Scientist; Bradley Jackson is a Resident; all at the University of New Mexico Health Sciences Center.

\section{Author disclosures}

The authors report no actual or potential conflicts of interest with regard to this article.

\section{Disclaimer}

The opinions expressed herein are those of the authors and do not necessarily reflect those of Federal Practitioner, Frontline Medical Communications Inc., the US Government, or any of its agencies. This article may discuss unlabeled or investigational use of certain drugs. Please review the complete prescribing information for specific drugs or drug combinations -including indications, contraindications, warnings, and adverse effects-before administering pharmacologic therapy to patients.

\section{References}

1. Wagner B, Kagan-Hallet KS, Russell IJ. Concomitant presentation of adermatopathic dermatomyositis, statin myopathy, fibromyalgia syndrome piriformis muscle myofascial pain and diabetic neuropathy. J Musculoskeletal Pain. 2003;11(2):25-30. doi:10.1300/J094v11n02_05

2. Collins R, Reith C, Emberson J, et al. Interpretation of the evidence for the efficacy and safety of statin therapy [published correction appears in Lancet. $2017 \mathrm{Feb}$ 11;389(10069):602]. Lancet. 2016;388(10059):25322561. doi:10.1016/S0140-6736(16)31357-5

3. Stroes ES, Thompson PD, Corsini A, et al Statinassociated muscle symptoms: impact on statin therapy-European Atherosclerosis Society Consensus Panel Statement on Assessment, Aetiology and Management. Eur Heart J. 2015;36(17):1012-1022. doi:10.1093/eurheartj/ehv043

4. Saku K, Zhang B, Noda K; PATROL Trial Investigators. Randomized head-to-head comparison of pitavastatin, atorvastatin, and rosuvastatin for safety and efficacy (quantity and quality of LDL): the PATROL trial. Circ J. 2011;75(6):1493-1505. doi:10.1253/circj.cj-10-1281

5. Wald JJ. The effects of toxins on muscle. Neurol Clin. 2000;18(3):695-718. doi:10.1016/s0733-8619(05)70219-x

6. Hoffman KB, Kraus C, Dimbil M, Golomb BA. A survey of the FDA's AERS database regarding muscle and tendon adverse events linked to the statin drug class. PLoS One. 2012;7(8):e42866. doi:10.1371/journal.pone.0042866

7. Sakaeda T, Kadoyama K, Okuno Y. Statin-associated muscular and renal adverse events: data mining of the public version of the FDA adverse event reporting system. PLoS One. 2011;6(12):e28124. doi:10.1371/journal.pone.0028124

8. Tiwari A. An overview of statin-associated proteinuria. Drug Discov Today. 2006;11(9-10):458-464. doi:10.1016/j.drudis.2006.03.017

9. Verhulst A, Sayer R, De Broe ME, D'Haese PC, Brown CD. Human proximal tubular epithelium actively secretes but does not retain rosuvastatin. Mol Pharmacol. 2008;74(4):1084-1091. doi:10.1124/mol.108.047647

10. Jones $\mathrm{PH}$, Davidson $\mathrm{MH}$, Stein $\mathrm{EA}$, et al. Comparison of the efficacy and safety of rosuvastatin versus atorvastatin, simvastatin, and pravastatin across doses (STELLAR* Trial). Am J Cardiol. 2003;92(2):152-160. doi:10.1016/s0002-9149(03)00530-7

11. Wanner C, Krane V, März W, et al. Atorvastatin in patients with type 2 diabetes mellitus undergoing hemodialysis [published correction appears in $N$ Engl J Med. 2005 Oct 13;353(15):1640]. N Engl J Med. 2005;353(3):238-248. doi:10.1056/NEJMoa043545

12. Fellström BC, Jardine AG, Schmieder RE, et al. Rosuvastatin and cardiovascular events in patients undergoing hemodialysis [published correction appears in $N$ Engl J Med. 2010 Apr 15;362(15):1450]. N Engl J Med. 2009;360(14):1395-1407. doi:10.1056/NEJMoa0810177

13. Holdaas H, Holme I, Schmieder RE, et al. Rosuvastatin in diabetic hemodialysis patients. J Am Soc Nephrol. 2011;22(7):1335-1341. doi:10.1681/ASN.2010090987

14. Naci H, Brugts JJ, Fleurence R, Tsoi B, Toor H, Ades AE. Comparative benefits of statins in the primary and secondary prevention of major coronary events and all-cause mortality: a network meta-analysis of placebo -controlled and active-comparator trials. Eur J Prev Cardiol. 2013;20(4):641-657. doi:10.1177/2047487313480435

15. Alsheikh-Ali AA, Ambrose MS, Kuvin JT, Karas RH. The safety of rosuvastatin as used in common clinical practice: a postmarketing analysis. Circulation. 2005;111(23):30513057. doi:10.1161/CIRCULATIONAHA.105.555482

16. Ward NC, Watts GF, Eckel $\mathrm{RH}$. Statin toxicity. Circ Res. $2019 ; 124(2): 328-350$. doi:10.1161/CIRCRESAHA.118.312782

17. Selva-O'Callaghan A, Alvarado-Cardenas M, PinalFernández I, et al. Statin-induced myalgia and myositis: an update on pathogenesis and clinical recommendations. Expert Rev Clin Immunol. 2018;14(3):215-224. doi:10.1080/1744666X.2018.1440206

18. Koslik $\mathrm{HJ}$, Meskimen $\mathrm{AH}$, Golomb BA. Physicians' Experiences as patients with statin side effects: a case series. Drug Saf Case Rep. 2017;4(1):3 doi:10.1007/s40800-017-0045-0 